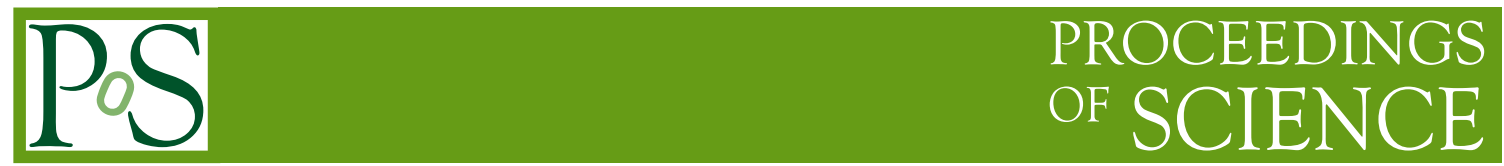

\title{
Jet tomography in hot QCD medium with deep learning
}

\author{
Yi-Lun Du, ${ }^{a, *}$ Daniel Pablos ${ }^{b}$ and Konrad Tywoniuk ${ }^{a}$ \\ ${ }^{a}$ Department of Physics and Technology, University of Bergen, \\ Postboks 7803, 5020 Bergen, Norway \\ ${ }^{b}$ INFN, Sezione di Torino, via Pietro Giuria 1, \\ I-10125 Torino, Italy \\ E-mail: yilun.du@uib.no, daniel.pablos.alfonso@to.infn.it, \\ Konrad. Tywoniuk@uib.no
}

With deep learning techniques, the degree of modification of energetic jets that traversed hot QCD medium can be identified on a jet-by-jet basis. Due to the strong correlations between the degree of jet modification and its traversed length in the medium, we demonstrate the power of our novel method to locate the creation point of a dijet pair in the nuclear overlap region. In particular, jet properties, such as jet width and orientation can serve as additional handles to locate the creation points to a higher level of precision, which constitutes a significant development towards the long-standing goal of using jets as tomographic probes of the quark-gluon plasma.

\footnotetext{
*** The European Physical Society Conference on High Energy Physics (EPS-HEP2021), *** *** 26-30 July $2021 * * *$

*** Online conference, jointly organized by Universität Hamburg and the research center DESY ***
}

\footnotetext{
${ }^{*}$ Speaker
} 


\section{Introduction}

Jets are narrow cones of hadrons that are produced in hard QCD processes in high-energy particle collisions. In heavy-ion collisions, they form concurrently with the creation of hot and dense QCD matter, known as the quark-gluon plasma (QGP), which behaves like a nearly perfect liquid. When passing through this medium, partonic jet modes will experience momentum diffusion and energy loss. The latter phenomenon, known as jet quenching, occurs by the radiation of soft particles towards large angles [1-4]. Unique properties of the medium are contained in the detailed modifications of these hard probes, which turn them into useful tools, and therefore tremendous theoretical and experimental effort is being devoted on them.

Using jets as differential probes of the evolution of the QGP created in heavy-ion collisions, aka jet tomography, is a long-term quest [5-10]. The medium-induced modifications of jets follow from the local properties of the medium along the jet trajectory. The capability to unambiguously capture the details of these interactions, for each individual jet, would lead to unprecedented precision in determining local properties of the fluid, including flow [11, 12], path-length dependence of jet modifications [13] and improved possibility of observing deconfined quasi-particles as degrees of freedom in the QGP [14-16].

In this paper we employ deep learning techniques to estimate the energy loss suffered by a given reconstructed jet at $p_{T}$ and cone size $R$ measured in a heavy-ion collision. Having this extracted knowledge at hand allows for many interesting applications, such as revealing more pronounced substructure modifications of jets [17], getting access to the genuine configuration profile of jets over the nuclear overlap region in the collision, both with respect to their creation points and orientations [18] and using jets as tomographic probes of the QGP, which is the main focus of this proceedings.

First, we demonstrate the power of our method to constrict the creation-point of a dijet pair over the nuclear overlap region by constraining the jet energy loss. Then, we present the improvement of the combination of the extraction of the lost energy with additional accessible knowledge about the jet width or the jet orientation with respect to the event plane of the collision. They allow to constrain the path length dependence separately for jets of specific width or jets traveling parallel and transverse to the event plane of the collisions, which refine the path to experimentally pinning down the original creation point of a dijet pair. These improvements contribute to the set of tools aimed at exploiting energetic jets as tomographic probes of the QGP.

\section{Jet energy loss with deep learning}

We first estimate, on a jet-by-jet basis, the amount of jet energy loss during the passage through a hot QCD medium, quantified through the variable $\chi=p_{T} / p_{T}^{\text {initial }}$ within the hybrid strong/weak coupling model [19-21]. $p_{T}$ is the measurable transverse momentum of a given jet in the presence of a medium, and $p_{T}^{\text {initial }}$ is the transverse momentum of the same jet would have had, had there been no medium. For further details on how to establish such a correspondence, see [17]. In the hybrid model, the vacuum evolution is factorized from the interactions with the medium. Other jet quenching models with this general picture should also allow for such a jet-by-jet correspondence $[17,18]$. 


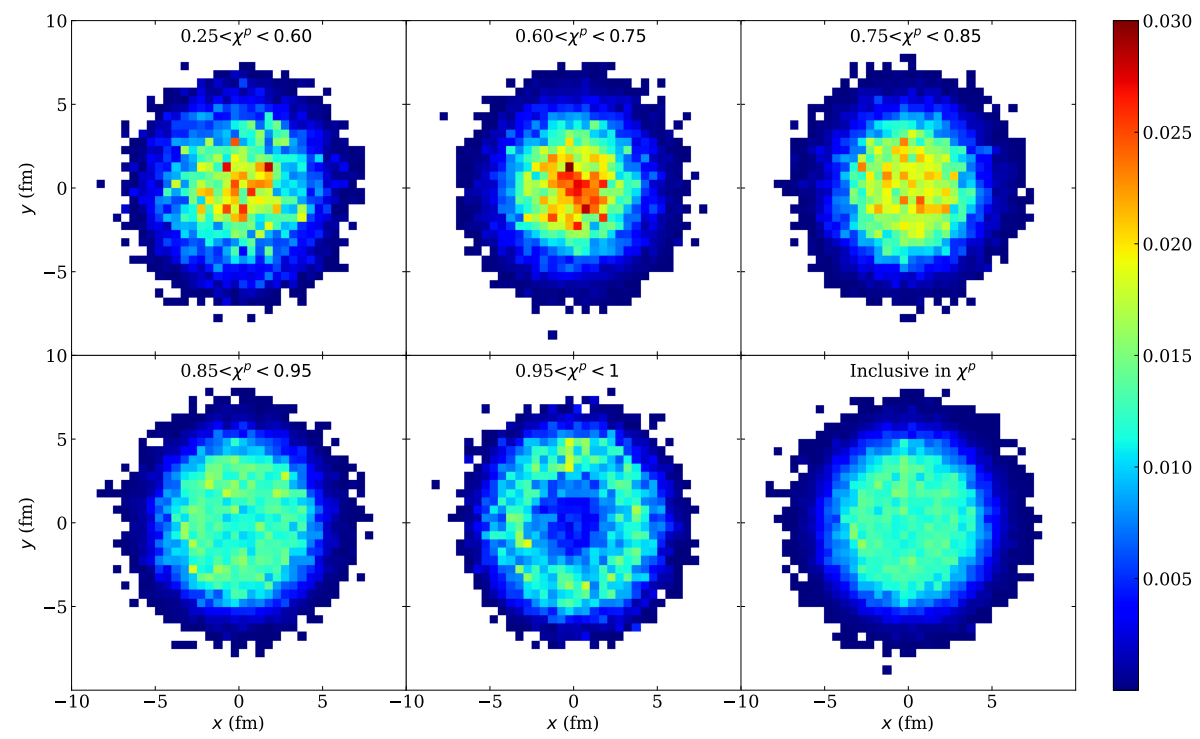

Figure 1: Creation point distributions of jets in the transverse plane for different ranges of the predicted $\chi^{p}$.

Within the hybrid model, we generate around 250,000 jets at $\sqrt{s_{N N}}=5.02 \mathrm{TeV}$ for $\mathrm{PbPb}$ collisions at 0-5\% centrality, which are reconstructed with FastJet 3.3.1 [22] using the anti- $k_{T}$ algorithm [23] with reconstruction parameter $R=0.4,|\eta|<2$ and measured $p_{T}>100 \mathrm{GeV}$. $80 \%$ of these samples are used for the training of our algorithm and the remaining $20 \%$ are used validation. We train the convolutional neural network $(\mathrm{CNN})$ to predict the energy loss ratio $\chi$ from the jet image in a supervised manner. The trained $\mathrm{CNN}$ can predict $\chi$ over a wide range with reasonable accuracy. For more details see Ref. [17].

\section{Jet tomography}

The traversed length by a given jet in QGP is strongly correlated to its energy loss and modifications. Specifically, by selecting jets suffering different amounts of energy loss $\chi$ extracted by our network, we are actually selecting jets traversing different lengths and consequently created at different positions in the transverse plane of the medium. This new capability paves the way to extract properties of QGP through jet tomographic applications with unprecedented precision. ${ }^{1}$

To corroborate this statement, we can look at the jet creation-points distribution in the transverse plane $\{x, y\}$ sliced in $\chi$, which is shown in Fig. 1. It's worth pointing out, again, that the pair values of creation positions $\{x, y\}$ are not extracted by our algorithm, but taken directly from the Monte Carlo instead. One can see that jets of the little quenched class, $0.95<\chi^{p}<1$, were created within a ring structure at the periphery of the nuclear overlap collision region. The creation points move towards the centre of the initial geometry gradually as energy loss increases. It's worth mentioning that for the most quenched class, $0.25<\chi^{p}<0.6$, the distribution becomes more spread than that

\footnotetext{
${ }^{1}$ Alternatively, the degree of medium induced jet modification can be reasonably estimated from the ratio of the jet $p_{T}$ over that of a recoiling colorless trigger boson, although their correlation is not tight due to sizeable out-of-cone radiation even in vacuum [8]. Besides, a recent tomographic study based on spatial-temporal gradient of QGP also gives clue to the creation point of an energetic jet [9].
} 


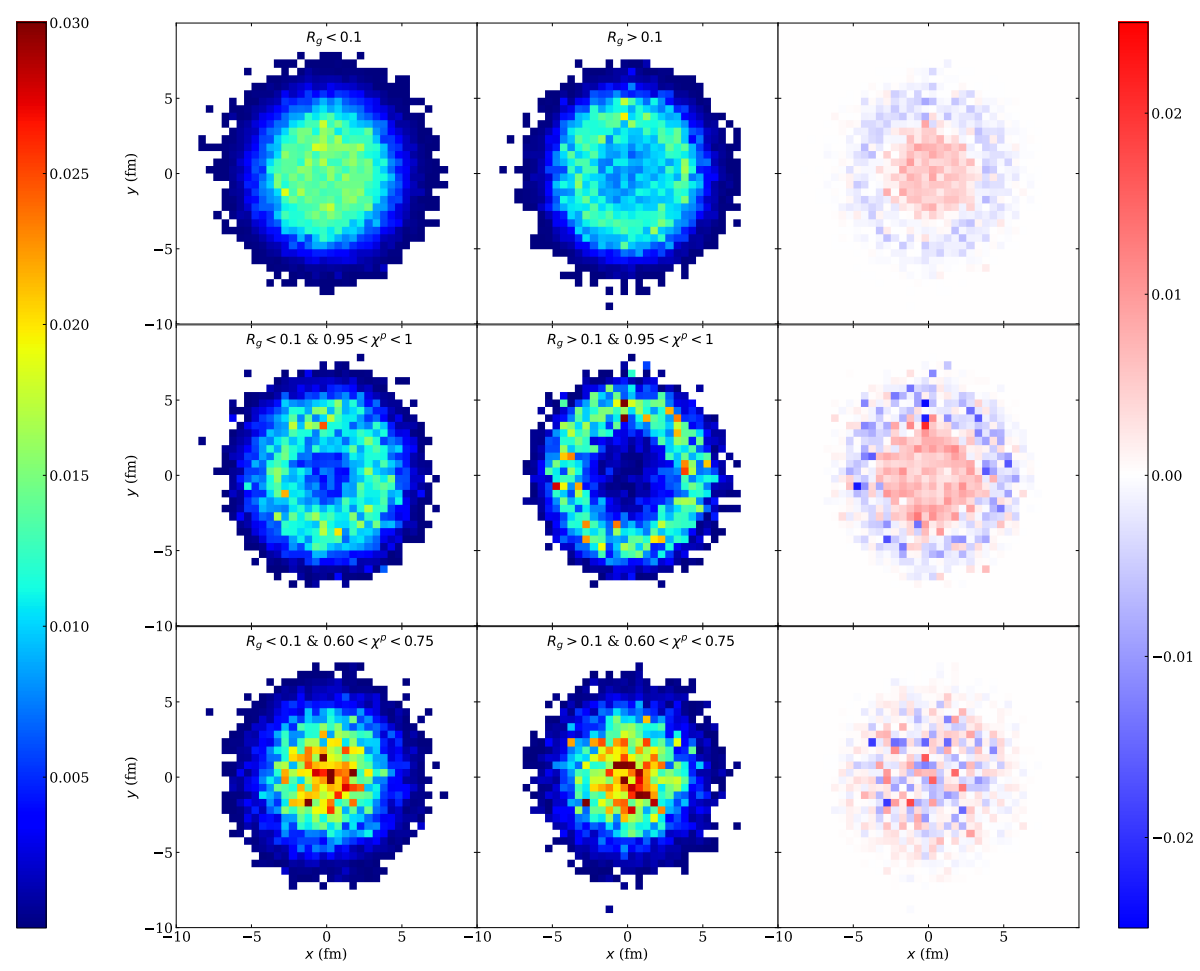

Figure 2: Creation point distributions in the transverse plane for jets with measured $R_{g}<0.1$ (left column) versus $R_{g}>0.1$ (middle column), and their difference (right column). Jets are inclusive in $\chi$ (upper row), in little quenched class with $0.95<\chi^{p}<1$ (middle row) and very quenched class with $0.6<\chi^{p}<0.75$ (lower row).

for the neighbour less quenched class, $0.6<\chi^{p}<0.75$. The reason can be attributed to that the most quenched jets have had to go through the largest traversed lengths, which correspond to jets that were created at the periphery and flew inwards, towards the center of the QGP. This observation inspires us to consider the jet orientation as an additional handle in a refined study. We will come back to this direction a bit later in the non-central collisions.

We have shown that with the extracted energy loss we have control of how long the jets have traversed within the QGP, which is an important step forward towards using jets as tomographic probes. One possible improvement of the above analysis could include more differential sample selections based on measurable jet properties, such as jet width $R_{g}$ within the Soft-Drop procedure [24], with parameters $z_{\text {cut }}=0.1$ and $\beta=0$. Given the dependence of energy loss on jet width, and its relation to selection bias, as discussed in Ref. [17], it is natural to expect that jets with different (initial) widths, and the same amount of the energy loss, will have traversed different lengths on average. To support this picture, we show in Fig. 2 the creation point distributions in the transverse plane for jets with a measured (this is, final) $R_{g}$ smaller or larger than 0.1 . In the top row (inclusive in $\chi$ ), we see that narrower jets tend to pass the selection even if they were created deep inside the medium, while wider jets are pushed towards the surface in comparison. The little quenched jets, $0.95<\chi^{p}<1$, (middle row) agree with this picture as the more pronounced ring structure shows. For the very quenched jets, $0.6<\chi^{p}<0.75$, one can see the reverse of such 

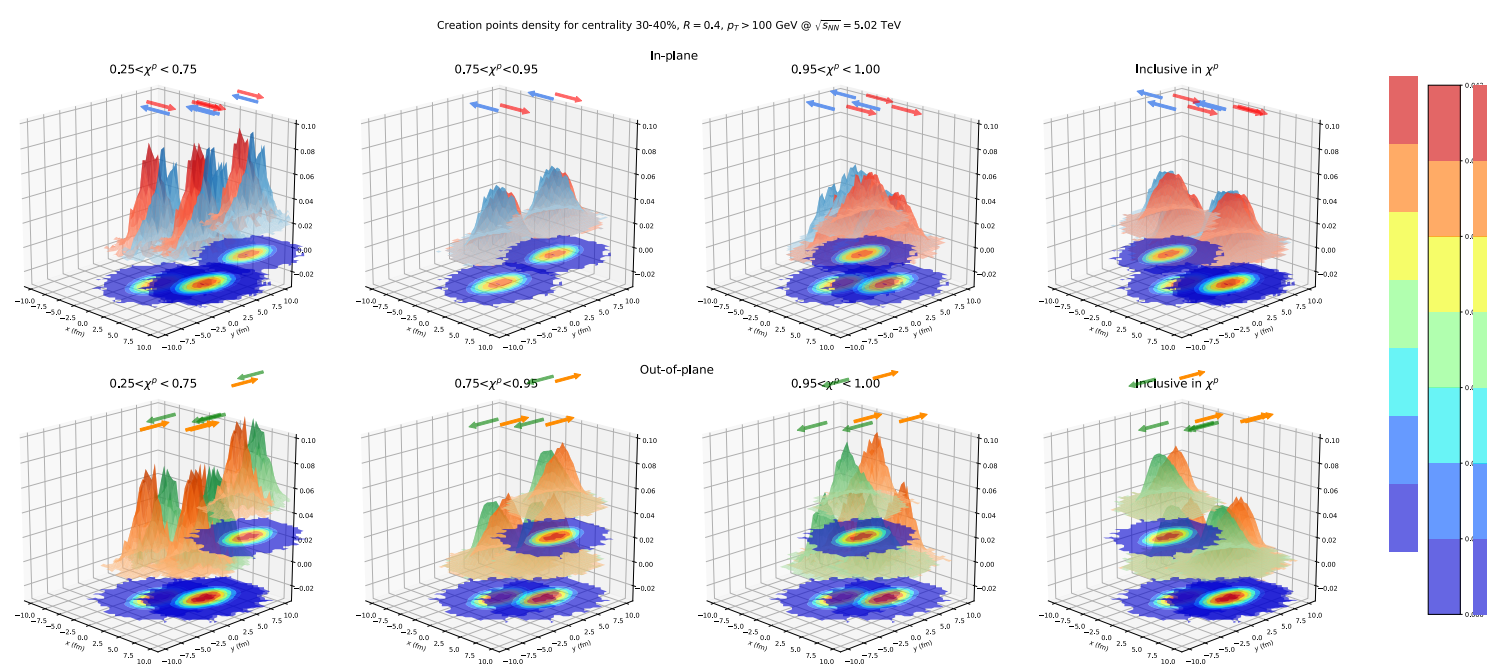

Figure 3: Creation point distributions of jets in the transverse plane in 30-40\% centrality for different ranges of the predicted $\chi^{p}$ in four columns, respectively. The in-plane jets propagating left (blue) and right (red) are shown in the upper row and the out-of-plane jets propagating up (orange) and down (green) are shown in the lower row. The 2-D histogram in the bottom of each plot is the distribution of the inclusive in-plane (upper row) and out-of-plane (lower row) jets in this centrality.

ordering as shown in the lower row, which is similar as in Fig. 1, since to get such quenched, narrow jets tend to be created towards the periphery and flow inwards.

To improve on locating the creation-point of a dijet pair in the nuclear overlap region more precisely, one could also account for the jet orientation with respect to the event plane of the collision, which is determined by the second azimuthal harmonic of the particle distribution. For demonstration purposes, we will consider jets propagating in-plane, i.e. parallel to the event plane, and out-of-plane, i.e. transverse to the event plane, generated at 30-40\% centrality at $\sqrt{s_{N N}}=5.02 \mathrm{TeV}$ for $\mathrm{PbPb}$ collisions instead. In Fig. 3 we show the results of the productionpoints distribution for around 900,000 jets. In the upper (lower) row we consider the jets that propagate in-plane (out-of-plane), which means they flow approximately along the short (long) axis of the nuclear overlap region. This corresponds to the jets with distinctly positive (negative) $v_{2}=\left(p_{T, x}^{2}-p_{T, y}^{2}\right) /\left(p_{T, x}^{2}+p_{T, y}^{2}\right)$. In the bottom of each sub-figure in the upper (lower) row we also show the production-point distribution of the in-plane (out-of-plane) jet inclusive in $\chi^{p}$. We can further select jets according to its propagation direction: either left (in blue) or right (in red) for the in-plane jets, and either up (in orange) or down (in green) for the out-of-plane jets. The histograms in each of fourth column show the results inclusive in $\chi^{p}$ and corresponds to the production-point distributions if we had no clue to the degree of jet energy loss. One can see some degree of separation, but the overlap is still quite large. This situation improves radically with the help of our knowledge of predicted $\chi^{p}$. The histograms in each of the first three columns display the production-point distributions for jets belonging to different quenching classes.

The third column of Fig. 3 shows results for little quenched jets, with $0.95<\chi^{p}<1$. To belong to this class, jets have to have traversed merely a short length through the QGP. Focusing on the out-of-plane jets first in the lower row, one can see that the production points of jets propagating 
upwards are predominantly localized in the upper hemisphere of the overlap region (and vice versa for jets propagating downwards). This reasoning also applies reversely for jets belonging to the very quenched class, with $0.25<\chi^{p}<0.75$, displayed in the first column. One can see that these very quenched jets propagating upwards have had to traverse a long length in the QGP, or analogously through a hot region. Consequently their production points will be predominantly localized in the lower hemisphere instead (and vice versa for jets propagating downwards). As the bridge between the little quenched and very quenched jets, the ones with $0.75<\chi^{p}<0.95$ shows the notably overlapping transition region in second column. Obviously, similar arguments could also apply for the in-plane jets displayed in the upper row of Fig. 3.

\section{Summary}

In this proceedings, we review the power of our novel deep learning techniques to locate with precision the jet creation point in the transverse plane, by selecting jets according to jet energy loss $\chi$, width $R_{g}$ and orientation, which constitutes a significant step towards the exploitation of tomographic power of energetic jets. In future work, the tomographic power is expected to be further improved by considering the interplay between the jet and the local properties of the medium, e.g., the local hydrodynamic flow $[11,12]$ or spatial-temporal gradients $[9,10,12]$, which determine preferred directions and deformed radiation spectra for the soft emissions from the jet. A direct extraction of the traversed length of energetic jets in the QGP is highly desirable to push forward this series of studies.

\section{Acknowledgments}

This work is supported by the Trond Mohn Foundation under Grant No. BFS2018REK01 and the University of Bergen. Y. D. thanks the support from the Norwegian e-infrastructure UNINETT Sigma2 for the data storage and HPC resources with Project Nos. NS9753K and NN9753K. D.P. has received funding from the European Union's Horizon 2020 research and innovation program under the Marie Skłodowska-Curie grant agreement No. 754496.

\section{References}

[1] D. d'Enterria, Landolt-Bornstein 23, 471 (2010), arXiv:0902.2011 [nucl-ex] .

[2] A. Majumder and M. Van Leeuwen, Prog. Part. Nucl. Phys. 66, 41 (2011), arXiv:1002.2206 [hep-ph] .

[3] Y. Mehtar-Tani, J. G. Milhano, and K. Tywoniuk, Int. J. Mod. Phys. A 28, 1340013 (2013), arXiv:1302.2579 [hep-ph] .

[4] J.-P. Blaizot and Y. Mehtar-Tani, Int. J. Mod. Phys. E 24, 1530012 (2015), arXiv:1503.05958 [hep-ph] .

[5] E. Wang and X.-N. Wang, Phys. Rev. Lett. 89, 162301 (2002), arXiv:hep-ph/0202105 . 
[6] T. Renk, Phys. Rev. C 74, 034906 (2006), arXiv:hep-ph/0607166.

[7] H. Zhang, J. F. Owens, E. Wang, and X.-N. Wang, Phys. Rev. Lett. 98, 212301 (2007), arXiv:nucl-th/0701045 .

[8] H. Zhang, J. F. Owens, E. Wang, and X.-N. Wang, Phys. Rev. Lett. 103, 032302 (2009), arXiv:0902.4000 [nucl-th] .

[9] Y. He, L.-G. Pang, and X.-N. Wang, Phys. Rev. Lett. 125, 122301 (2020), arXiv:2001.08273 [hep-ph] .

[10] W. Chen, Z. Yang, Y. He, W. Ke, L.-G. Pang, and X.-N. Wang, Phys. Rev. Lett. 127, 082301 (2021).

[11] N. Armesto, C. A. Salgado, and U. A. Wiedemann, Phys. Rev. C 72, 064910 (2005), arXiv:hep$\mathrm{ph} / 0411341$.

[12] A. V. Sadofyev, M. D. Sievert, and I. Vitev, (2021), arXiv:2104.09513 [hep-ph] .

[13] B. Betz and M. Gyulassy, JHEP 08, 090 (2014), [Erratum: JHEP 10, 043 (2014)], arXiv:1404.6378 [hep-ph] .

[14] F. D’Eramo, K. Rajagopal, and Y. Yin, JHEP 01, 172 (2019), arXiv:1808.03250 [hep-ph] .

[15] J. Barata, Y. Mehtar-Tani, A. Soto-Ontoso, and K. Tywoniuk, Phys. Rev. D 104, 054047 (2021).

[16] M. Cè, T. Harris, H. B. Meyer, and A. Toniato, JHEP 03, 035 (2021), arXiv:2012.07522 [hep-ph] .

[17] Y.-L. Du, D. Pablos, and K. Tywoniuk, JHEP 21, 206 (2020), arXiv:2012.07797 [hep-ph] .

[18] Y.-L. Du, D. Pablos, and K. Tywoniuk, Phys. Rev. Lett. 128, 012301 (2022).

[19] J. Casalderrey-Solana, D. C. Gulhan, J. G. Milhano, D. Pablos, and K. Rajagopal, JHEP 2015, 175 (2015).

[20] J. Casalderrey-Solana, D. C. Gulhan, J. G. Milhano, D. Pablos, and K. Rajagopal, JHEP 03, 053 (2016), arXiv:1508.00815 [hep-ph] .

[21] J. Casalderrey-Solana, D. Gulhan, G. Milhano, D. Pablos, and K. Rajagopal, JHEP 03, 135 (2017), arXiv:1609.05842 [hep-ph] .

[22] M. Cacciari, G. P. Salam, and G. Soyez, Eur. Phys. J. C 72, 1896 (2012), arXiv:1111.6097 [hep-ph] .

[23] M. Cacciari, G. P. Salam, and G. Soyez, JHEP 04, 063 (2008), arXiv:0802.1189 [hep-ph] .

[24] A. J. Larkoski, S. Marzani, G. Soyez, and J. Thaler, JHEP 05, 146 (2014), arXiv:1402.2657 [hep-ph] . 